\title{
Pulmonary fibrosis and lung cancer: not the same disease, but not so different
}

\author{
Sebastiano Emanuele Torrisi ${ }^{1}$ \\ Silvia Puglisi ${ }^{1}$ \\ Virginia Vindigni ${ }^{1}$ \\ Riccardo Giuliano ${ }^{1}$ \\ Stefano Palmucci² \\ Carlo Vancheri ${ }^{1}$
}

\begin{abstract}
1 Regional Centre for Interstitial and Rare Lung Diseases, Department of Clinical and Experimental Medicine, University Hospital Policlinico-Vittorio Emanuele, Catania, Italy

2 Radiodiagnostic and Radiotherapy Unit, University Hospital Policlinico-Vittorio Emanuele, Catania, Italy
\end{abstract}

\section{Address for correspondence:}

Sebastiano Emanuele Torrisi, MD

Regional Centre for Interstitial and Rare Lung Diseases

Department of Clinical and Experimental Medicine University Hospital Policlinico-Vittorio Emanuele

Via Santa Sofia 78 - 95123 Catania, Italy

Phone: +390953781774 - Fax: +390953781468

E-mail: torrisiseby@hotmail.it

\section{Summary}

Idiopathic pulmonary fibrosis (IPF) is a chronic, progressive, fibrosing interstitial pneumonia characterized by a poor survival, even worse than many cancers. Recent studies have demonstrated that IPF and cancer share several cellular and molecular alterations related to epigenetic and genetic changes, altered regulation of apoptosis, abnormal response to regulatory signals, abnormal expression of microRNAs (mRNAs), reduced cell-to-cell communication and activation of specific signaling pathways. This leads to the hypothesis that IPF can be considered, in some respects, a cancer-like disease. This correlation may help in understanding the pathogenesis of IPF by exploiting the great knowledge of the biological mechanisms studied in cancer but it may also help in increasing the awareness of this disease at public, political and even at healthcare level. In addition, the identification of common pathogenic pathways between the two diseases may stimulate new clinical trials with cancer drugs, as in the case of nintedanib, and drugs combinations or different lines of drugs as largely experimented in cancer.
KEY WORDS: interstitial lung diseases, idiopathic pulmonary fibrosis, cancer, epigenetic alterations, cell to cell communications, signal transduction pathways, myofibroblasts, TGF- $\beta$, tyrosine kinases.

\section{Introduction}

Idiopathic pulmonary fibrosis (IPF) is defined as a "specific form of chronic, progressive, fibrosing interstitial pneumonia of unknown cause, occurring in adults, limited to the lungs and associated with the histopathological and/or radiological pattern of usual interstitial pneumonia" (1). Incidence and prevalence have not been easy to esti-
IPF may be considered in some respects a cancer-like disorder of the lung. mate because previous studies have only recently had a uniform definition of IPF. The annual incidence of IPF in the USA is estimated at 6.8 to 16.3 per 100,000 (2), while in Europe there is not a single global value but only data from individual countries (3).

For a long time, IPF was considered the result of a chronic inflammatory process of the lung caused by an unknown agent. However, this hypothesis has been discarded because IPF is characterized from early phase by the presence of variable degree of fibrosis with mild or no inflammation at histology (4). This supports the idea that there are two pathways that lead to fibrosis: the "inflammatory route" and the "epithelial-fibroblastic route" (5). The first one, common to non-IPF interstitial lung diseases (ILDs), is characterized by an early phase of alveolitis accompanied by the recruitment and activation of inflammatory cells which in turn may activate lung fibroblasts, ultimately causing fibrosis. The second route is typical of IPF. Here, damaged alveolar epithelial cells without the participation of inflammatory cells, are able to stimulate the migration and activation of mesenchymal cells via the production of a number of growth factors and chemokines, leading to the formation of aggregates of fibroblasts and abnormal deposition of collagen and extracellular matrix components. This abnormal repairing process is driven by a series of pathogenic events that are described in other chronic degenerative diseases and interestingly also in cancer. Some authors have in effect defined cancer as a wound that does not heal with a series of other analogies with IPF including the etiology, which is often unknown, similar risk factors such as smoking and/or environmental or professional exposure, and the presence of a specific genetic background considered im- 
portant for the occurrence of the disease. InterestingIy, IPF and cancer have in common a series of cellular and molecular alterations related to epigenetic and genetic changes, altered regulation of apoptosis, abnormal response to regulatory signals, abnormal expression of microRNAs (mRNAs), reduced cell-to-cell communication and activation of specific signaling pathways. Based on this evidence we have hypothesized that IPF may be considered in some respects a cancer-like disorder of the lung. The purpose of this short review is to analyse the main reasons supporting the concept of IPF as a cancer-like disease $(6,7)$.

\section{Epigenetic and genetic alterations}

Epigenetic disorders are alterations by which the environment alters the degree of activity of genes without changing the DNA sequences and the information contained therein. Therefore, epigenetic alterations

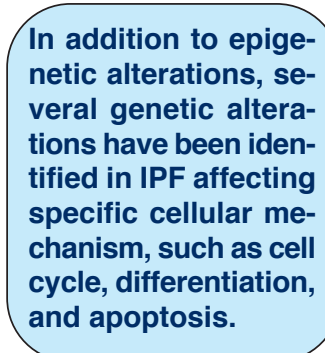
are related to aging, cigarette smoke, nutritional, chemical and physical factors. It has been recently shown that such alterations are involved not only in the initiation and progression of cancer but also in the pathogenesis of IPF $(8,9)$. In cancer, the most frequent epigenetic disorders are represented by methylation of suppressor genes and hypomethylation of oncogenes. According to Rabinowich, similar alterations may be present in IPF. Thy- 1 is a glycoprotein expressed by normal fibroblasts, where the hypermethylation of its promoter region causes the reduction of the expression of the related Thy-1 glycoprotein (10). The loss of expression of this glycoprotein facilitate the differentiation of fibroblasts into myofibroblasts $(11,12)$. Similarly, in cancer, the same alteration is associated with a more invasive behavior of the neoplasia. In addition to epigenetic alterations, several genetic alterations have been identified in IPF affecting specific cellular mechanisms, such as cell cycle, differentiation and apoptosis. Mutation of p53 and fragile histidine triade (FHIT) as well as micro-satellite instability and loss of gene heterozygosity are very often distinctive of many cancers. Interestingly in IPF, these alterations are present in about $50 \%$ of IPF patients and in most of the cases observed in the parenchymal areas of honeycombing, the paradigmatic lesion of IPF (13-15). Another common feature of cancer is the altered expression of telomerase and the subsequent shortening of telomeres. Telomerase is a reverse transcriptase ribonucleoprotein that protects chromosomes from degradation, irregular recombination and end-to-end fusions $(16,17)$. Recently, Antoniou et al. have demonstrated a lower expression of two subunits of telomerase (hTERT and $h$-TERC mRNA) in lung tissue from IPF patients compared with lung tissue of controls and even from patients with non-small cell lung cancer (16). An important pathogenic role in both cancer and IPF is also played by microRNAs (miR). They are non coding small RNA molecules involved in post transcriptional gene expression, regulating different molecular and cellular processes. It has been demonstrated that aberrant expression of $\mathrm{miR}$ is linked to initiation and progression of cancer (18). The miR-200 family, also inhibits TGF- $\beta 1$ induced-EMT (epithelial mesenchymal transition), a process through which an epithelial cell switches its features in a mesenchymal cell. In addition, in the bleomycin mouse model of fibrosis, miR-200 as well as MiR-29 are down-regulated suggesting an important new pathogenic role for these molecules (19). It has also been shown that some genes associated with the fibrotic phenotype were up-regulated after the inhibition of miR-29 in human fetal lung fibroblasts (20). Pandit et al. showed that TGF- $\beta$ down-regulates let-7d expression and SMAD3 binding to the let-7d promoter. Inhibition of let-7d causes increases in mesenchymal markers $\mathrm{N}$-cadherin-2, vimentin, and a-smooth muscle actin (ACTA2) as well as HMGA2 in epithelial cell lines (21). Furthermore, the use of an "antagomir" specific for the let-7 family results in the down-regulation of epithelial markers suggesting an important role for the let-7 family in lung fibrosis. In IPF there is also an upregulation of miR-21 in myofibroblasts. This has been well demonstrated in lungs of mice treated with bleomycin and in lungs of patients with IPF. MiR-21 normally promotes TGF- $\beta$ signaling by inhibiting Smad7 amplifying the TGF- $\beta$ pathway. However, expression changes in microRNA-21 and the role of miR-21 in epithelial-mesenchymal transition during lung fibrosis have not yet been completely defined (22). Pottier et al. have also demonstrated increased expression of miR-155 in lungs of mice with bleomycin-induced fibrosis as well as in IPF lungs. The up-regulation of miR-155 leads to the down-regulation of a number of target genes mediating fibroblast migration (23). From these studies, the observation that miRs expression in IPF lung correlates with disease severity also emerges, as demonstrated by the findings of increased levels of miRNAs (miR-302c, miR-423, miR-210, miR-376c, and miR-185) in lung biopsies of rapidly progressing patients with IPF (24).

\section{Altered cell to cell communications}

Cells continuously interact through junctional communication to coordinate normal tissue functions, to maintain homeostasis and to obtain a synchronized tissue behavior. Gap junctions are specialized intercellular connections formed by two connexons composed by hexameric oligomers of transmembrane proteins called connexins. Gap junctions have a critical role in the lungs. They regulate secretion of pulmonary surfactant mediated by alveolar cell and prevent lung injury by improving barrier function. Some studies have shown that inhibition of gap junctions cause a barrier function reduction in mouse models developing 
symptoms similar to pulmonary fibrosis $(25,26)$. Cancer is characterized by a loss of cell to cell communication mediated by gap junction and by a reduction in connexins expression suggesting that in order to progress, tumor cells need to isolate themselves from the influence of surrounding normal cells. Lower levels of
The loss of proliferative control in IPF cells could be caused by an altered fibroblast to fibroblast communication mediated by a reduced expression of connexin 43 , very similar to what happens in cancer.
Cx43 have been identified for example in lung cancer and in gastric cancer cells (27). Cx43 is also involved in the wound-healing of the skin. In this process cell to cell communication and gap junctions have an important role in modulating inflammation and tissue repair. It has been demonstrated that the down regulation of Cx43 protein foster the proliferation and migration of keratinocytes and fibroblasts (28). We have hypothesized that the loss of proliferative control in IPF cells could be caused by an altered fibroblast to fibroblast communication mediated by a reduced expression of connexin 43 , very similar to what happens in cancer. Effectively, we have demonstrated that IPF fibroblasts, as observed in cancer cells, have a reduced ability to express connexin 43 (29).

\section{Uncontrolled proliferation: the role of myofibrob- lasts}

One of the distinctive characteristics of IPF is the presence in lung biopsy of fibroblastic foci, formed by myofibrobasts whose origin is still unclear. Three different theories have been proposed to explain their

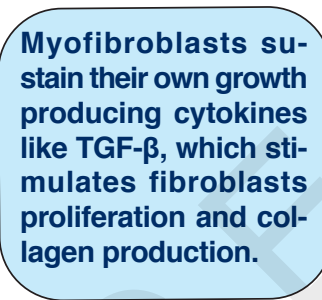
origin. According to the first, myofibroblasts arise from resident fibroblasts and organize themselves into fibrolastic foci, producing excessive amounts of extracellular matrix (ECM) proteins. It has been supposed that injured alveolar cells produce mediators responsible for migration of local fibroblasts to the injured site and for their differentiation in myofibroblasts (30).

The second hypothesis describes myofibroblasts as derived from resident epithelial cells through a process of migration and cellular differentiation called epithelial mesenchymal transition (EMT). Epithelial cells lose cell to cell attachment and adhesion molecules like E-caderin, reorganize cytoskeleton and assume mesenchymal markers like fibronectin and alfasmooth-muscle actin. This transition is a form of metaplasia and is very similar to what happens in cancer (31). It has been hypothesized that EMT also contribute to cancer progression and metastasis. This process is supported by extracellular factors like TGF$\beta$, growth factors and metalloproteinases that promote cancer progression. TGF- $\beta$ is also produced by can- cer cells and stimulates fibroblasts differentiation and migration to the cancer site.

According to the third hypothesis, myofibroblasts derive from circulating fibrocytes originated from bone marrow and express typical markers of mesenchymal cells such as fibronectin (32). It has been shown that some of the myofibroblasts in the tumor stroma originate from circulating fibrocytes. Consequently, they have an important function in cancer development. Cancer associated fibroblasts produce different mediators, such as metalloproteinases and growth factors to support their own growth. Metalloproteinases are a group of zinc-dependent endopeptidases, which act by destroying extracellular matrix and remodeling tissues. In cancer their role is to break down connective tissue barriers permitting cancer cells to infiltrate the surrounding tissues (33). This is very similar to what happens in IPF: myofibrobasts sustain their own growth producing cytokines like TGF- $\beta$, which stimulates fibroblasts proliferation and collagen production. Cancer progression is also facilitated by the expression of some molecules, such as laminin, heat shock proteins and fascin. Laminin is an extracellular matrix protein (expressed on the basement membrane) and it is overexpressed at the invasive front of different cancers (34). Laminin stimulates cancer progression and many studies have shown that laminin high expression is linked to a more invasive behaviour of cancer. Heat shock protein (HSP) are a family of proteins whose overexpression is linked to cancer capacity of metastasize. HPS are involved in many cellular processes such as protein folding and are a powerful regulator of cell apoptosis, that interacts with specific signaling pathways. In particular, HP 27 expressed in cancer cells is associated with a poor prognosis and high capacity to metastasize. Fascin is usually not expressed in normal epithelial cells but it is present in human carcinoma. Many studies have indicated fascin as a candidate biomarker for aggressive carcinomas (35). In IPF, epithelial cells surrounding fibroblasts foci also express fascin, HSP 27 and laminin suggesting, once again, an important similarity between IPF cells and cancer cells (36).

\section{Activation of signal transduction pathways}

A large variety of signal transduction pathways are activated both in cancer and in IPF and many studies have also demonstrated their involvement in the pathogenesis of these two diseases. The Wnt signaling pathways are a group of signal transduction pathways made up of proteins that pass signals from the outside of a cell, through cell surface receptors, to the inside of the cell. It has been demonstrated that the alterations of this pathway may have clinical relevance in a variety of diseases, including lung cancer, mesothelioma and
Failure of cell apoptosis represents a key process in carcinogenesis and in chemoresistance mechanisms in cancer but it is also present in IPF. 
desmoid tumors (37). The Wnt/ $\beta$-catenin signaling pathway regulates the expression of some molecules involved in tissue invasion such as matrilysin, laminin and cyclin-D1 and it is also involved in a biologically relevant crosstalk with TGF- $\beta$. It is well known that $W n t / \beta$-catenin pathway is strongly activated in IPF lung tissue as evidenced by extensive nuclear accumulation of $\beta$-catenin at different involved sites such as bronchiolar proliferative lesions, damaged alveolar structures and fibroblast foci (38). The functional significance of $\beta$-catenin has been well demonstrated by the presence of an intense immunoreactivity for $\beta$-catenin and by the expression of high levels of cyclin-D1 and matrilysin. We have also demonstrated that Wnt/ $\beta$-catenin pathway may also be activated by TGF- $\beta$ through the induction of alpha-smooth actin (SMA) expression via extracellular-regulated kinases (ERK)1/2 activation, glycogen synthase kinase-3beta (GSK-3beta) inhibition and nuclear $\beta$-catenin translocation that leads to fibroblast activation and collagen production in human lung fibroblasts (39).

Failure of cell apoptosis represents a key process in carcinogenesis and in chemo-resistance mechanisms in cancer but it is also present in IPF. In this regard, the PI3K/Akt signaling pathway plays an important role. This pathway is involved in the process of cell growth, proliferation and survival. Phosphoinositol-3kinase (PI3K) stimulates the synthesis of phosphatidylinositol-3,4,5-triphosphate (PIP3) which causes the activation of Akt regulating cellular processes such as protection from apoptosis (40). The PI3K family is divided into three different classes: Class I, Class II, and Class III based on primary structure, regulation and in vitro lipid substrate specificity. Recently, we assessed the expression of class I PI3K p110 isoforms in IPF lung tissue as well as in tissue-derived fibroblast cell lines evaluating the effect of the selective inhibition of p110 isoforms on IPF fibroblast proliferation and fibrogenic activity. The expression of PI3K p110 $\alpha$, $\beta$ and $\gamma$ isoforms does not differ between normal and IPF tissue/tissue-derived fibroblasts whereas $\mathrm{p} 110 \gamma$ was more expressed in both IPF lung homogenates and ex vivo fibroblast cell lines with a strong immunoreactivity for $\mathrm{p} 110 \gamma$. Furthermore, both $\mathrm{p} 110 \gamma$ pharmacological inhibition and gene silencing were able to significantly inhibit proliferation rate as well as a-SMA expression in IPF fibroblasts. This suggests that PI3K p110 $\gamma$ isoform may have an important role in the pathogenesis of IPF and can be a specific pharmacological target (41). In this regard, a recent study has demonstrated that oral administration of p110 $\gamma$ inhibitor prevents bleomycin-induced pulmonary fibrosis in rats (42). More recently researchers have focused on another signal transduction pathway in IPF: the JAK-STAT signaling pathway. This system is a major signaling alternative to the second messenger system such as Cyclic AMP, Cyclic GMP, Inositol Triphosphate, Diacylglycerol and Calcium. It transmits information from chemical signals outside the cell, through the cell membrane, and into gene promoters on the DNA in the cell nucleus, which causes DNA transcription and activity in the cell. JAK-STAT signaling path- way is frequently altered in many cancers. One of the regulatory mechanism of JAK-STAT signaling pathway is represented by the SOCS family (suppressor of cytokine signaling proteins). The role of SOCS1 in IPF pathogenesis has been recently studied by Bao et al. According to this author, a lower expression of SOCS1 was identified in IPF patients and this finding has been related to severe manifestations of disease and to a worse prognosis (43).

Another key signaling pathway strongly activated in many cancers but also in IPF is represented by tyrosine kinases pathway. Tyrosine kinases catalyze the phosphorylation of tyrosine residues in some proteins. They are involved in cell growth, differentiation, adhesion, motility and regulation of cell death. Mutations can make tyrosine kinases a nonstop functional state. This may lead to initiation or progression of cancer. An important activity of this pathway has been also studied in wound healing and fibrogenesis $(44,45)$. Platelet-derived growth factor (PDGF), a ligand of tyrosine kinase receptor, is a potent growth factor for fibroblasts in vitro and some fibrogenic mediators like TGF- $\beta$ and basic fibroblast growth factor (FGF) have PDGF-dependent profibrotic activities. There is evidence that PDGF protein and mRNA are increased in IPF. In particular high levels of PDGF (A-D) isoforms have been shown in irradiated mice and the use of a PDGF receptor inhibitor has attenuated the development of pulmonary fibrosis in animal models induced by radiation $(46,47)$. As well as PDGF, fibroblast growth factor (FGF) is largely involved not only in carcinogenesis but also in fibrogenesis. FGF receptors are present on epithelial cell and fibroblasts and mediate EMT and fibroblast transition into myofibroblasts (48). The use of tyrosine kinase receptors inhibitors have been widely used in non small cell lung carcinoma and other cancers. These growth factors have potent mitogenic effects, so their inhibition is expected to reduce fibrosis in IPF, as already happens in cancer therapy where they are widely used. Imatinib-mesylate, a specific PDGFr inhibitor, was evaluated for its potential antifibrotic effects in preclinical and clinical studies. It was able to inhibit fibroblast proliferation and collagen deposition in vitro and in vivo but when it was evaluated in a clinical trial, no benefits in slowing progression were found $(49,50)$. Triple inhibition seems to be associated to a more potent antifibrotic effect. On this basis, BIBF 1000, an inhibitor of PDGFr, VEGFr and FGFr, was evaluated in a mice model of bleomycin-induced pulmonary fibrosis and in an ex vivo fibroblast differentiation assay. It was found to attenuate fibrogenesis by reducing the expression of pro fibrogenetic factors and by decreasing collagen deposition (51). On the same line, BIBF 1120 (nintedanib), a triple kinase inhibitor with potent suppressing effects on VEGFr, PDGFr and FGFr, was also evaluated. More recently, nintedanib was studied in few clinical trials as a potential antifibrotic therapy in IPF demonstrating that treatment with this drug may reduce of about $50 \%$ the decline in lung function of IPF patients. Based on these results, nintedanib has been approved as a new and additional therapeutic 
possibility together with pirfenidone in patients with IPF (52-54).

\section{Conclusions}

In spite of the great progress observed in diagnosing, managing and treating IPF during the past few years, the disease is still marked by a disappointing survival and the diagnosis is too often made late when the disease is already in advanced stages. Worsening the situation is the lack of valid diagnostic and/or prognostic biomarkers and above all the poor awareness of this disease at public, political and even at the healthcare levels. The awareness of cancer as a potentially fatal disease is instead well understood and the need for supporting cancer research well established at any level
The idea of IPF as a cancer-like disease burdened by a survival even worse than many cancers may help in increasing the awareness of this disease. of the public opinion. In virtue

of this, during the last few years we have witnessed to progressive improvement in the diagnostic and therapeutic strategies against cancer. The idea of IPF as a cancer-like disease burdened by a survival even worse than many cancers may help in increasing the awareness of this disease. In addition the identification of common pathogenic pathways between the two diseases may stimulate new clinical trials with cancer drugs, as in the case of nintedanib, and hopefully with combinations or different lines of drugs as largely experimented in cancer.

\section{References}

1. Raghu G, Collard HR, Egan JJ, Martinez FJ, Behr J, Brown KK, et al. An official ATS/ERS/JRS/ALAT statement: idiopathic pulmonary fibrosis: evidencebased guidelines for diagnosis and management. Am J Respir Crit Care Med. 2011;183:788-824.

2. Raghu G, Weycker D, Edelsberg J, Bradford WZ, Oster $\mathrm{G}$. Incidence and prevalence of idiopathic pulmonary fibrosis. Am J Respir Crit Care Med. 2006;174:810-816.

3. Thomeer M, Demedts M, Vandeurzen K. Registration of interstitial lung diseases by 20 centres of respiratory medicine in Flanders. Acta Clin Belg. 2001; 56:163-172.

4. Idiopathic Pulmonary Fibrosis Clinical Research Network, Raghu G, Anstrom KJ, King TE Jr, Lasky JA, Martinez FJ. Prednisone, Azathioprine, and N-Acetylcysteine for Pulmonary Fibrosis. N Engl J Med. 2012;366:1968-77.

5. Selman M, Pardo A. Idiopathic pulmonary fibrosis: an epithelial/fibroblastic cross-talk disorder. Respir Res. 2002;3:3

6. Kim KK, Kugler MC, Wolters PJ, Robillard L, Galvez MG, Brumwell AN, et al. Alveolar epithelial cell mesenchymal transition develops in vivo during pul- monary fibrosis and is regulated by the extracellular matrix. Proc Natl Acad Sci USA. 2006;103:1318013185.

7. Vancheri C, Failla M, Crimi N, Raghu G. Idiopathic pulmonary fibrosis: a disease with similarities and links to cancer biology. Eur respir J. 2010;35:496504.

8. Nakamura J, Tanaka T, Kitajima $\mathrm{Y}$, Noshiro $\mathrm{H}$, Miyazaki K. Methylation-mediated gene silencing as biomarkers of gastric cancer: A review. World J Gastroenterol. 2014;20(34):11991-12006.

9. Ling Q, Shi W, Huang C, Zheng J, Cheng Q, Yu K et al. Epigenetic silencing of dual oxidase 1 by promoter hypermethylation in human hepatocellular carcinoma. Am J Cancer Res. 2014;4(5):508-17.

10. Rabinovich EI, Kapetanaki MG, Steinfeld I, Gibson KF, Pandit KV, Yu G, et al. Global methylation patterns in idiopathic pulmonary fibrosis. PLoS One. 2012;7:e33770.

11. Sanders YY, Kumbla P, Hagood JS. Enhanced myofibroblastic differentiation and survival in Thy-1(-) lung fibroblasts. Am J Respir Cell Mol Biol. 2007; 36:226-235.

12. Sanders YY, Pardo A, Selman M, Nuovo GJ, Tollefsbol TO, Siegal GP, et al. Thy-1 promoter hypermethylation: a novel epigenetic pathogenic mechanism in pulmonary fibrosis. Am J Respir Cell Mol Biol. 2008; 39:610-618.

13. Hojo S, Fujita J, Yamadori I, Kamei T, Yoshinouchi T, Ohtsuki Y, et al. Heterogeneous point mutations of the p53 gene in pulmonary fibrosis. Eur Respir J. 1998;12:1404-1408.

14. Uematsu K, Yoshimura A, Gemma A, Mochimaru H, Hosoya $Y$, Kunugi S, et al. Aberrations in the fragile histidine triad (FHIT) gene in idiopathic pulmonary fibrosis. Cancer Res. 2001;61:8527-8233.

15. Demopoulos K, Arvanitis DA, Vassilakis DA, Siafakas NM, Spandidos DA. MYCL1, FHIT, SPARC, P16 (INK4) and TP53 genes associated to lung cancer in idiopathic pulmonary fibrosis. J Cell Mol Med. 2002; 6:215-222.

16. Antoniou KM, Samara KD, Lasithiotaki I, et al. Differential telomerase expression in idiopathic pulmonary fibrosis and non-small cell lung cancer. Oncolog Rep. 2013;30(6):2617-2624.

17. Calado RT, Young NS. Telomere diseases. N Engl J Med. 2009;361(24): 2353-2365.

18. Vancheri C. Common pathways in idiopathic pulmonary fibrosis and cancer. Eur Respir Rev. 2013; 22:265-272.

19. Yang S, Banerjee S, de Freitas A, Sanders YY, Ding $Q$, Matalon S, et al. Participation of miR-200 in pulmonary fibrosis. Am J Pathol. 2012;180(2):484-493.

20. Cushing L, Kuang PP, Qian J, Shao F, Wu J, Little F, et al. miR-29 is a major regulator of genes associated with pulmonary fibrosis. Am J Respir Cell Mol Biol. 2011;45:287-94.

21. Pandit KV, Corcoran D, Yousef $H$, Yarlagadda $M$, Tzouvelekis A, Gibson KF, et al. Inhibition and role of let- $7 d$ in idiopathic pulmonary fibrosis. Am J Respir Crit Care Med. 2010;182:220-9.

22. Yamada M, Kubo H, Ota C, Takahashi T, Tando Y, 
Suzuki T, et al. The increase of microRNA-21 during lung fibrosis and its contribution to epithelial-mesenchymal transition in pulmonary epithelial cells. Respir Res. 2013;14:95.

23. Pottier N, Maurin T, Chevalier B, Puisségur MP, Lebrigand $\mathrm{K}$, Robbe-Sermesant $\mathrm{K}$, et al. Identification of keratinocyte growth factor as a target of microRNA-155 in lung fibro- blasts: implication in epithelial-mesenchymal interactions. PLoS One. 2009; 4:e6718.

24. Oak SR, Murray L, Herath A, Sleeman M, Anderson I, Joshi AD, et al. A micro RNA processing defect in rapidly progressing idiopathic pulmonary fibrosis. PLoS One. 2011;6:e21253.

25. Kojima T, Spray DC, Kokai Y, Chiba H, Mochizuki Y, Sawada N. Cx32 formation and/or Cx32-mediated intercellular communication induces expression and function of tight junctions in hepatocytic cell line. Exp Cell Res. 2002;276(1):40-51.

26. Koval M, Billaud M, Straub AC, Johnstone SR, Zarbock A, Duling BR, et al. Spontaneous lung dysfunction and fibrosis in mice lacking connexin 40 and endothelial cell connexin 43. Am J Pathol. 2011; 178(6):2536-2546.

27. Fallon RF, Goodenough DA. Five-hour half life of mouse liver gap-junction protein. J Cell Biol. 1981;90 (2):521-526.

28. Mori R, Power KT, Wang CM, Martin P, Becker DL. Acute downregulation of connexin43 at wound sites leads to a reduced inflammatory response, enhanced keratinocyte proliferation and wound fibroblast migration. J Cell Sci. 2006;119(Pt 24):5193-203.

29. Trovato-Salinaro A, Trovato-Salinaro E, Failla M, Mastruzzo C, Tomaselli V, Gili E, et al. Altered intercellular communication in lung fibroblast culture from patients with IPF. Respir Res. 2006;7:122.

30. Selman M, Pardo A. Role of epithelial cells in idiopathic pulmonary fibrosis: from innocent targets to serial killers. Proc Am Thorac Soc. 2006;3(4):364-372.

31. Willis BC, Liebler JM, Luby-Phelps K, Nicholson AG, Crandall ED, du Bois RM, et al. Induction of epithelial-mesenchymal transition in alveolar epithelial cells by transforming growth factor-beta: potential role in idiopathic pulmonary fibrosis. Am J Pathol. 2005;166 (5):1321-1332.

32. Andersson-Sjöland A, de Alba CG, Nihlberg K, Becerril C, Ramírez R, Pardo A, et al. Fibrocytes are a potential source of lung fibroblasts in idiopathic pulmonary fibrosis. Int J Biochem Cell Biol. 2008;40 (10):2129-2140.

33. Dancer RC, Wood AM, Thickett DR. Metalloproteinases in idiopathic pulmonary fibrosis. Eur Respir J. 2011;38(6):1461-1467.

34. Niki T, Kohno T, Iba S, Moriya Y, Takahashi Y, Saito $\mathrm{M}$, et al. Frequent co-localization of Cox-2 and laminin-5 gamma2 chain at the invasive front of early-stage lung adenocarcinomas. Am J Pathol. 2002;160(3):1129-1141.

35. Hashimoto Y, Skacel M, Adams JC. Roles of fascin in human carcinoma motility and signaling: prospects for a novel biomarker? Int J Biochem Cell Biol. 2005;37(9):1787-804.
36. Chilosi M, Zamò A, Doglioni C, Reghellin D, Lestani $M$, Montagna $L$, et al. Migratory marker expression in fibroblast foci of idiopathic pulmonary fibrosis. Respir Res. 2006;7:95.

37. Mazieres J, He B, You L, Xu Z, Jablons DM. Wnt signaling in lung cancer. Cancer Lett. 2005;222(1):1-10.

38. Chilosi M, Poletti V, Zamò A, Lestani M, Montagna L, Piccoli $\mathrm{P}$, et al. Aberrant Wnt/beta-catenin pathway activation in idiopathic pulmonary fibrosis. Am J Pathol. 2003;162(5):1495-1502.

39. Caraci F, Gili E, Calafiore M, Failla M, La Rosa C, Crimi N, et al. TGF-beta1 targets the GSK-3beta/ beta-catenin pathway via ERK activation in the transition of human lung fibroblast into myofibroblasts. Pharmacol Res. 2008;57(4):274-282.

40. Cantley LC. The phosphoinositide 3-kinase pathway. Science. 2002;296(5573):1655-7.

41. Conte E, Gili E, Fruciano M, Korfei M, Fagone E, lemmolo $\mathrm{M}$, et al. PI3K p110g overexpression in idiopathic pulmonary fibrosis lung tissue and fibroblast cells: in vitro effects of its inhibition. Lab Invest. 2013;93:566-576.

42. Wei X, Han J, Chen ZZ, Qi BW, Wang GC, Ma YH, et al. A phosphoinositide 3-kinasegamma inhibitor, AS605240 prevents bleomycin- induced pulmonary fibrosis in rats. Biochem Biophys Res Commun. 2010;397:311-317.

43. Bao Z, Zhang Q, Wan H, He P, Zhou X, Zhou M. Expression of suppressor of cytokine signaling 1 in the peripheral blood of patients with idiopathic pulmonary fibrosis. Chin Med J. 2014;127(11):2117-20.

44. Beyer C, Distler JH. Tyrosine kinase signaling in fibrotic disorders: translation of basic research to human disease. Biochim Biophys Acta. 2013;1832:897904.

45. Hetzel M, Bachem M, Anders D, Trischler G, Faehling M. Different effects of growth factors on proliferation and matrix production of normal and fibrotic human lung fibroblasts. Lung. 2005;183:225-237.

46. Antoniades HN, Bravo MA, Avila RE, Galanopoulos T, Neville-Golden J, Maxwell M, et al. Platelet-derived growth factor in idiopathic pulmonary fibrosis. J Clin Invest. 1990;86:1055-1064.

47. Abdollahi A, Li M, Ping G, Plathow C, Domhan S, Kiessling $F$, et al. Inhibition of platelet-derived growth factor signaling attenuates pulmonary fibrosis. J Exp Med. 2005;201:925-935.

48. Chaudhary NI, Roth GJ, Hilberg F, Müller-Quernheim J, Prasse A, Zissel G, et al. Inhibition of PDGF, VEGF and FGF signalling attenuates fibrosis. Eur Respir J. 2007;29:976-985.

49. Vuorinen K, Gao F, Oury TD, Kinnula VL, Myllarniemi M. Imatinibmesylate inhibits fibrogenesis in asbestosinduced interstitial pneumonia. Exp Lung Res. 2007;33:357-373.

50. Daniels CE, Lasky JA, Limper AH, Mieras K, Gabor E, Schroeder DR. Imatinib treatment for idiopathic pulmonary fibrosis: Randomized placebo-controlled trial results. Am J Respir Crit Care Med. 2010; 181:604-610.

51. Chaudhary NI, Roth GJ, Hilberg F, Muller-Quernheim J, Prasse A, Zissel G, et al. Inhibition of pdgf, 
vegf and fgfsignalling attenuates fibrosis. Eur Respir J. 2007;29:976-985.

52. Richeldi L, Costabel U, Selman M, Kim DS, Hansell DM, Nicholson AG, et al. Efficacy of a tyrosine kinase inhibitor in idiopathic pulmonary fibrosis. N Engl J Med. 2011;365:1079-1087.

53. Brown KK RL, Costabel U, Flaherty KR, Kim D, Noble PW, Raghu G, et al. Treatment of IPF with the ty- rosine kinase inhibitor bibf 1120: Patient-reported outcomes in the tomorrow trial. Am J Respir Crit Care Med. 2012;185:A3634.

54. Richeldi L, Cottin V, Flaherty KR, Kolb M, Inoue Y, Raghu G, et al. Design of the INPULSIS ${ }^{\mathrm{TM}}$ trials: two phase 3 trials of nintedanib in patients with idiopathic pulmonary fibrosis. Respir Med. 2014;108(7):102330. 